\title{
Comparative morphometry of the temporomandibular joint in brachycephalic and mesocephalic cats using multislice $\mathrm{CT}$ and cone beam CT
}

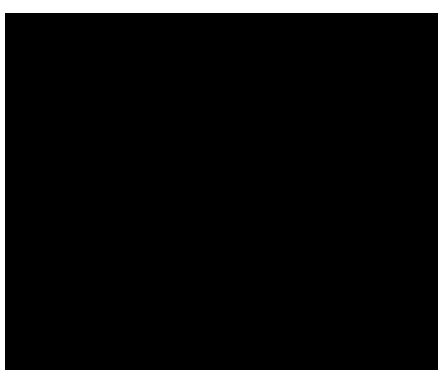

\author{
Quentin Delesalle ${ }^{1}$, António M Lopes², Jerzy P Gawor ${ }^{3}$, \\ Davide Zani ${ }^{4}$, Hugo M Pereira5(D) and Lisa A Mestrinho2 ${ }^{2}$
}

\begin{abstract}
Objectives The purpose of this study was to analyse and compare morphometric measurements of the temporomandibular joint (TMJ) in two groups of cats with brachycephalic and mesocephalic craniofacial skull conformations using multislice CT and cone beam CT.

Methods CT records of 20 cats without TMJ pathology were included in the study, and were divided into two groups according to those with mesocephalic or brachycephalic skull conformation. Width and depth of the mandibular fossa, width and height of the head of the mandible, and two different angles were measured in 40 TMJs.

Results TMJ conformation differed between groups: $3 / 6$ parameters were statistically different. Brachycephalic cats had a significantly narrower mandibular fossa, a shorter head of the mandible and a wider angle of congruence (angle 2).

Conclusions and relevance The results provide morphometric measurements that can serve as a reference tool for the veterinary radiologist and surgeon when TMJ evaluation is necessary. Moreover, the study identified significant differences between the two craniofacial skull conformations.
\end{abstract}

Keywords: Temporomandibular joint; morphometry; computed tomography; cone beam computed tomography; brachycephalic; mesocephalic

\section{INTRODUCTION}

Multislice CT (MSCT) is considered superior to conventional radiography to evaluate the bony com-

ponents of the temporomandibular joint (TMJ). ${ }^{1,2}$

MSCT and cone beam CT (CBCT) provide excel- lent bone detail, spatial orientation and modern post- processing algorithms, which allow three-dimensional, sagittal and dorsal reconstructions. ${ }^{1-4}$ Nevertheless, artic- ular soft tissue structures, such as articular cartilage, artic- ular discs and joint capsules, cannot be fully evaluated.5,6

Brachycephalic breeds differ from domestic short- hair (DSH) cats in various craniofacial features. In pre- vious studies using CT, brachycephalic cats presented a dorsally flat skull, with a brachygnathic maxilla, an aberrant occlusal pattern and deviation of the maxil- lary canine tooth, leading to obstruction of the ventral nasal passage. ${ }^{7,8}$ Moreover, different authors suggest a

reports provide a low level of evidence to corroborate this theory. ${ }^{2,9}$ predisposition of brachycephalic cats to open-jaw lock- ing and possible TMJ dysplasia, although a couple of

The objectives of this study were to characterise the morphology of the TMJ in a group of cats by reporting morphometric measurements and angles of joint con- gruence using MSCT and CBCT, and to compare these parameters between brachycephalic and mesocephalic skull conformations.

\section{Animals}

MSCT and CBCT scans of the head of cats older than

1 year, which included the TMJ area (from the dor-sal articular eminence of the mandibular fossa to the occipital condyle), were selected from the records of three veterinary clinical centres. None of the animals had a history of TMJ disorder or other disease with an impact on the TMJ such as dental disease, tumours or fractures. Also, there were no signs of degenerative changes detected in any examination. Moreover, studies were selected and then separated based on breed, and brachycephalic (traditional Persian) and mesocephalic (DSH) conformation. 
The cranial index - the ratio of cranial width to cra- nial length - was determined in mesocephalic cats in order to confirm the absence of brachycephalic features $(\leqslant 0.6)$, as previously described. ${ }^{8}$ The cranial index was not performed for brachycephalic cats because they were all purebred Persian cats with obvious brachycephalic features.

All selected studies were retrospectively analysed, having been performed with clinical indication and under the highest standard of good care. Informed consent was obtained to access the CT images for the study.

\section{CT assessment}

Equipment used for image acquisition was as follows:

16-slice helical CT scanner (HiSpeed LX/i; General Electric Company, Medical Systems, EUA and Toshiba Astelion, Canon Medical Systems Europe BV) and a CBCT scanner (Newtom 5G XL Vet; CEFLA). Scan parameters are given in Table 1. Image analysis, axial, sagittal and coronal reconstructions were obtained using a DICOM reader software (Osirix Imaging Software; Pixmeo).

Both the MSCT and CBCT scanners employed in this study are subjected to periodic maintenance to ensure image quality, namely isotropic spatial resolution of both acquisition and reconstruction of volumetric datasets. This includes routine calibrations where standard phan- toms are employed in test scans to confirm the geometric accuracy of all measurements.

\section{Procedures}

All veterinary centres performed CT scans with the ani- mals under general anaesthesia, positioned routinely in sternal recumbency with the ventral borders of the mandi- ble in the horizontal plane and the mouth partially opened by the presence of the endotracheal tube. If the mandible could not be positioned in a horizontal plane naturally, a cushion was used to obtain the best positioning.

DICOM images retrieved were imported into the soft- ware. The axis of image acquisition was verified before identification of the anatomical landmarks; if necessary, minimal adjustments were performed using the three- dimensional multiplanar reconstruction software modal- ity. The axis was considered adequate if the condylar processes of the mandibles were visible and symmetri- cal in axial reconstruction and if the supramastoid crests were visible and symmetrical in coronal reconstruction (Figures 1 and 2).

The anatomical landmarks used to determine morphometry of the TMJ were the mandibular fossa, the maximal ventral extension of the retroarticular process, the dorsal articular eminence of the mandibular fossa, the medial and lateral extremities of the head of the mandible, and the supramastoid crest. These anatomical landmarks were used to identify the estimated axis of rotation of the condylar process (EARCP) adapted from a similar study in the dog (Figure 1). ${ }^{10}$ The supramastoid crest was always the first to be identified and marked with a dot, using the three-dimensional surface rendering option in the software (Figure 2a). The left and right supramastoid crests were subsequently visualised on the coronal reconstruction and aligned with the axial axis (Figure 2b). Alignment was confirmed with the visualisation of the two dots in the axial reconstruction (Figure 2c). The other landmarks were subsequently selected in the sagit- tal reconstruction in which the supramastoid crest mark was transected (Figure 1b).
The EARCP corresponded to the centre of a circle drawn at the sagittal reconstruction transected by the supramastoid crest, over the mandibular condylar pro-cess, being carefully adapted to the articular contour, according to a previous report. ${ }^{10}$ Two angles (1 and 2 ) were determined.

Anlge 1 was calculated using the following lowing anatomical points: the maximal lateral extension of the supramastoid crest, the EARCP and the maximal ventral extension of the retroarticular process (Figure 3a). This angle reflected the degree of the ventral extension of the retroarticular process in relation to the articular head of the condylar process. Angle 2 was based on the dorsal articular eminence of the mandibular fossa, the EARCP and the maximal ventral extension of the retroarticular process (Figure 3b). Joint congruence was appreciated, from a geometric perspective, using angles 1 and 2 from EARCP, which reflects the TMJ's capability to distribute an applied load.

The width and depth of the mandibular fossa were determined using the sagittal CT reconstruction. The width corresponded to a straight line drawn from the maximal ventral extension of the retroarticular process to the dorsal articular eminence of the mandibular fossa. The depth corresponded to the perpendicular distance from the midpoint of the first line to the subchondral bone of the mandibular fossa (Figure 4).

The length and height of the caudal portion of the condylar process of the mandible (head of the mandible) corresponded, first, to the longest distance from the two extremes of the condylar process in parallel to the man- dibular fossa (Figure 5); and, second, as the perpendicular measurement to the previous line passing through the EARCP marked in the axial projection (Figure 5).

The width and depth of the mandibular fossa, and angles 1 and 2 were determined in the sagittal reconstruc- tion and length and height of the head of the mandible in the axial reconstruction.

Each TMJ was evaluated separately $(n=40)$. All measurements, except for the angles, were registered in millimetres. Each variable was initially measured three times in different periods in time by the same observer (QD).

\section{Statistical analysis}

All variables were registered in a commercial software calculator package (Microsoft Excel version 16.28 for $\mathrm{MAC}$ ), and then imported into an open-source statisti- cal software package ( $\mathrm{R}$ version 3.5.1). For each continu- ous variable, each TMJ was evaluated separately and the median, minimum and maximum value and SD for each TMJ were calculated. Cohen's kappa (K) test was used to assess intraobserver agreement. The Shapiro-Wilk test was used for each variable to confirm normality and an independent samples $t$-test was performed for compari- son among variables.

\section{RESULTS}

Twenty cats were included in the study: nine were female $(45 \%)$, and 11 were male $(55 \%)$. Ten were DSH cats with a mean age of 5.5 years (range 1-13 years) and a mean body weight of $4.95 \mathrm{~kg}$ (range $3.40-6.50 \mathrm{~kg}$ ). There were 10

Persians with a mean age of 5.7 years (range $1-10$ years) and a mean body weight of $4.50 \mathrm{~kg}$ (range $3-7.20 \mathrm{~kg}$ ).

Both TMJs were assessed in each cat, resulting in 40 TMJs being evaluated. The intra-observer agreement was excellent $(K=0.82)$. The mean width and depth of the 
mandibular fossa in this group of 20 cats were, respec- tively, $5.55 \mathrm{~mm}$ and $1.77 \mathrm{~mm}$. The mean of angles 1 and

2 were $63.87^{\circ}$ and $148.19^{\circ}$, respectively. The mean length and height of the head of the mandible were $12.89 \mathrm{~mm}$ and 4.23 $\mathrm{mm}$, respectively. Comparison of the TMJ CT parameters is given in Table 2 . All variables measured were normally distributed. The width of the mandibular fossa, the head of the mandible height and angle 2 were significantly different between DSH and Persian cats $(P=0.0039, P=0.0078$ and $P$ $=0.031$, respectively). Brachycephalic cats had a significantly narrower mandibular fossa, a shorter head of the mandible and a wider angle of congruence (angle 2) (Figure 6).

\section{DISCUSSION}

This study describes the morphometric features of the feline TMJ using MSCT and CBCT. TMJ physiological angles and measurements have been shown to be useful in humans in the diagnosis and development of models, surgical plan- ning and design of prosthetic devices. ${ }^{11}$

In this study the measurements were performed using MSCT and CBCT images, two systems that differ in methodology. Although veterinary studies evaluating anatomi- cal accuracy and comparison between methods (MSCT and $\mathrm{CBCT}$ ) are lacking, their reconstruction algorithms provide isometrically similar and accurate images for bone, as demonstrated in previous studies in the human field. . $^{3,4}$

The morphometric data documented in this study iden- tified differences in the conformation of the mandibular fossa, in the head of the mandible and in joint congru- ity between two groups of cats with different skull con- formations. The findings were of statistical significance, despite of the small number of individuals, which was a limitation of the study. Other limitations of the study were the absence of the inclusion of the dolichocephalic skull type conformation verified in the Siamese breed.

The morphometric description of the TMJ in the cat adapted a previously described work performed in the dog. ${ }^{10}$ In our work, the supramastoid crest was used as an anatomical landmark instead of the nuchal crest, as used in the dog, ${ }^{10}$ because it was not consistently observed in all sagittal slices in the cat. The supramastoid crest is a strongly marked prolongation of the squamous part of the temporal bone, and runs backwards from the upper border of the zygomatic process, above the external acoustic meatus. ${ }^{12}$

In dogs, mesocephalic and brachycephalic skull types also showed important differences in the TMJ, and con- formations associated with specific breeds were also encountered. ${ }^{10}$ Although cats do not present as much phenotypical variability as is observed in dogs, the dif- ferences reported here were consistent.

This study found significant differences between the conformation of the condylar process of the mandible, mandibular fossa and angle. Brachycephalic cats have a significantly narrower mandibular fossa compared with mesocephalic cats; however, the depth of the mandibu- lar fossa was not significantly different. The head of the mandible was significantly shorter in the brachycephalic group compared with the mesocephalic group, whereas the length was not significantly different. Angle 1, which quantifies the ventral extension of the retroarticular pro- cess over the head of the mandible, ${ }^{10}$ was not significantly different between the two groups. However, angle 2, which reflects the congruence between the articular head of the condylar process and the mandibular fossa of the temporal bone, was wider in the brachycephalic group. Angle 2 is related to the depth of the mandibular fossa and the degree of ventral extension of the retroarticular process (angle 1). As these last two parameters (depth of the mandibular fossa and angle 1) were not significantly different between the groups, it is expected that a higher value of angle 2 corresponds to a more profound insertion of the head of the mandible onto the mandibular fossa. Given the semi-conic shape of the condylar process of the mandible and the convex-shaped mandibular fossa, these differences reflect that there is a less developed condylar process in brachycephalic cats, but that it is congruent in a less developed mandibular fossa.

To our knowledge, there are no studies associating skull conformations and TMJ disease in the cat. Some authors suggest a higher predisposition for luxation in brachycephalic cats than in other breeds. ${ }^{11,13,14}$ Luxation, ${ }^{13}$

TMJ dysplasia ${ }^{9}$ and open-mouth jaw locking ${ }^{15,16}$ have all been described in Persian cats. Some hypotheses sug- gested in these papers included abnormal masticatory forces due to malocclusion, symphyseal and TMJ lax-ity, pterygoid muscle spasm and TMJ dysplasia. $2,5,11,13-16$

Regardless of the proposed theories, TMJ conformational differences were not previous identified by these authors. Moreover, the anatomical differences observed in this study should be considered of potential clinical impor- tance as they could contribute to the suggested predispo- sition of brachycephalic cats to TMJ luxation.

More studies enrolling a larger sample of mesoce-phalic and brachycephalic cats, as well as including doli- chocephalic breeds, are necessary in order to evaluate whether distinctive anatomical differences do contribute to TMJ disease.

\section{CONCLUSIONS}

This study described the tomographic morphometry of the TMJ in domestic cats and showed differences between two skull conformations (mesocephalic and brachy-cephalic). The brachycephalic group had a narrower mandibular fossa and a shorter mandible head than the mesocephalic group. So far, no parameter or measure- ment has suggested a particular predisposition of TMJ diseases in brachycephalic cat breeds, but anatomical TMJ differences, identified here, should be considered in future studies looking at TMJ luxation and openjaw locking.

Acknowledgements The authors thank Ricardo Santos for his contribution to the image editing, and Telmo Nunes for statistical assistance.

Author note The preliminary results were published, in Eng- lish, as part of a dissertation research project by QD, supervised by DZ and LAM. This paper was presented in poster form at the 28th European Veterinary Dental Forum Utrech, The Neth- erlands, May 2019.

Conflict of interest The authors declared no potential conflicts of interest with respect to the research, authorship, and/or publication of this article.

Funding This work was supported by the Project UIDP/ CVT/00276/2020 (funded by FCT).

Ethical approval This work involved the use of nonexperimental animals only (including owned or unowned animals and data from prospective or retrospective studies). Established 
internationally recognised high standards ('best practice') of individual veterinary clinical patient care were followed. Ethical approval from a committee was therefore not necessarily required.

Informed consent Informed consent (either verbal or writ- ten) was obtained from the owner or legal custodian of all animal(s) described in this work (either experimental or non- experimental animals) for the procedure(s) undertaken (either prospective or retrospective studies). No animals or humans are identifiable within this publication, and therefore addi- tional informed consent for publication was not required.

\section{References}

1 Bar-Am Y, Pollard RE, Kass PH, et al. The diagnostic yield of conventional radiographs and computed tomography in dogs and cats with maxillofacial trauma. Vet Surg 2008; 37: 294-299.

2 Beam RC, Kunz DA, Cook CR, et al. Use of three-dimensional computed tomography for diagnosis and treatment plan- ning for open-mouth jaw locking in a cat. $J$ Am Vet Med Assoc 2007; 230: 59. DOI: 10.2460/javma.230.1.59.

3 Matta RE, von Wilmowsky C, Neuhuber W, et al. The impact of different cone beam computed tomography and multi-slice computed tomography scan parameters on virtual threedimensional model accuracy using a highly precise exvivo evaluation method.J Craniomaxillofac Surg 2016; 44: 632-636.

4 Leung CKK, Pow EHN, Li TKL, et al. Accuracy of radiographic measurements for implant planning using cone- beam and helical computer tomography. J Investig Clin Dent 2017; 8. DOI: $10.1111 /$ jicd.12232.

5 Schwarz T, Weller R and Dickie AM. Imaging of the canine and feline temporomandibular joint: a review. Vet Radiol Ultrasound 2002; 43: 85-97.

6 Arzi B, Cissell DD, Verstraete FJM, et al. Computed tomographic findings in dogs and cats with temporomandib- ular joint disorders: 58 cases (2006-2011). $J$ Am Vet Med Assoc 2013; 242: 69-75.

7 Schlueter C, Budras DK, Ludewig E, et al. Brachycephalic feline noses: CT and anatomical study of the relationship between head conformation and the nasolacrimal drain- age system. $J$ Feline Med Surg 2009; 11: 891-900.

8 Schmidt MJ, Kampschulte M, Enderlein S, et al. The relationship between brachycephalic head features in modern Persian cats and dysmorphologies of the skull and inter- nal hydrocephalus. J Vet Intern Med 2017; 31: 1487-1501.

9 Nutt EA, Anderson T, Gracis M, et al. Open-mouth jaw locking in cats: a literature review and use of computed tomography in three cases. J Feline Med Surg 2018; 20: 1180-1191.

10 Villamizar-Martinez AL, Cristian MV, Marco AG, et al. Morphologic and morphometric description of the temporomandibular joint in the domestic dog using com-puted tomography. $J$ Vet Dent 2016; 33: 75-82.

11 Coombs MC, Bonthius DJ, Nie X, et al. Temporomandibu- lar joint condyle-disc morphometric sexual dimorphisms independent of skull scaling. J Oral Maxillofac Surg 2019; 77: 42-53

12 Konig HE and Liebich H-G. Veterinary anatomy of domes- tic mammals: textbook and colour atlas. 3rd ed. New York: Schattauer, 2007.

13 Oakes MG, Lewis DD and Pechman RD. Intermittent open mouth locking in a Persian cat. Vet Comp Orthop Traumatol 1990; 3: 97-99.

14 Soukup JW, Snyder CJ and Gengler WR. Computed tomography and partial coronoidectomy for open-mouth jaw locking in two cats. $J$ Vet Dent 2009; 26: 226-233.

15 Lantz GC. Intermittent open-mouth locking of the temporomandibular joint in a cat. $J$ Am Vet Med Assoc 1987; 190: 1574

16 Reiter AM. Symphysiotomy, symphysiectomy, and intermandibular arthrodesis in a cat with open-mouth jaw locking: case report and literature review. J Vet Dent 2004; 21: $147-158$. 


